\title{
Isomorphism Classes of Maximal Intersecting Uniform Families Are Few
}

\author{
Geoffrey McKenna \\ Department of Mathematics \\ The George Washington University \\ Washington, D.C. 20052, USA \\ gmckenna@gwu.edu
}

Submitted: May 24, 2004; Accepted: Nov 14, 2005; Published: Nov 29, 2005

Keywords: maximal intersecting; uniform antichains

\begin{abstract}
Denote by $f(k, m)$ the number of isomorphism classes of maximal intersecting $k$-uniform families of subsets of $[m]$. In this note we prove the existence of a constant $f(k)$ such that $f(k, m) \leq f(k)$ for all values of $m$.
\end{abstract}

\section{Introduction}

Let $m$ be a natural number. By $[m]$ we mean the set of integers $\{1,2, \ldots m\}$. A family $\mathcal{F}$ of subsets of $[m]$ is said to be $k$-uniform if every member of $\mathcal{F}$ has $k$ elements. In symbols, this is sometimes (as in, e.g., $[1,5]$ ) written as $\mathcal{F} \subseteq[m]^{(k)}$ or $\mathcal{F} \subseteq\left(\begin{array}{c}{[m]} \\ k\end{array}\right)$; in what follows we adopt the first of these. $\mathcal{F}$ is said to be intersecting if the intersection of any two of its members is nonempty. An intersecting $k$-uniform family $\mathcal{F}$ is said to be maximal if it is maximal with respect to its being an intersecting $k$-uniform family. Two families $\mathcal{F}_{1}$ and $\mathcal{F}_{2}$ are said to be isomorphic if each can be obtained from the other by permuting the ground set $[m]$.

Lemma 1.1. The number of pairwise nonisomorphic intersecting $k$-uniform families grows monotonically with $m$, and is at least $\left(\begin{array}{c}m-1 \\ k-1\end{array}\right)$.

Proof. We construct a collection $S$ of $\left(\begin{array}{c}m-1 \\ k-1\end{array}\right)$ pairwise nonisomorphic families. There are $\left(\begin{array}{c}m-1 \\ k-1\end{array}\right)$ subsets of $[m]$ containing the element 1 . Order these at whim. Let $\mathcal{F}_{j}$ consist of the the first $j$ of these for $j \in\left\{1,2, \ldots\left(\begin{array}{c}m-1 \\ k-1\end{array}\right)\right\}$. Each family $\mathcal{F}_{j}$ is $k$-uniform and intersecting, since any two sets in $\mathcal{F}_{j}$ contain the common element 1 . Since the families $\mathcal{F}_{i}$ and $\mathcal{F}_{j}$ have different cardinality for $i \neq j$, they are nonisomorphic. 
The purpose of this trivial lemma is as follows: If we replace the phrase "pairwise nonisomorphic intersecting $k$-uniform families" by "pairwise nonisomorphic maximal intersecting $k$-uniform families", we get a different, perhaps surprising, answer:

Theorem 1.2. The number of pairwise nonisomorphic maximal intersecting $k$-uniform families of subsets of $[m]$ is bounded by a constant $f(k)$ not depending on $m$.

The next section introduces the chief device by which we treat these problems. Section Three presents our proofs. The concluding section offers some conjectures on how the bounds given here can be improved.

\section{The Pedestal of a $k$-Uniform Family}

Definition 2.1. Given a family $\mathcal{F} \subseteq[m]^{(k)}$, define its pedestal $P(\mathcal{F}) \subseteq 2^{[m]}$ to be the collection of those minimal subsets $S$ of $[m]$ satisfying the following rules:

$i$ To avoid trivialities, $|S| \leq k$.

ii Every $k$-superset of $S$ is in $\mathcal{F}$.

Note that the members of $\mathcal{F}$ satisfy both rules i and ii, but are typically not minimal with respect to this property.

Here are a couple of examples of pedestals:

Example 2.2. For $m \geq 2 k$, the Erdös-Ko-Rado theorem states that a maximum interesecting family $\mathcal{F} \subseteq[m]^{(k)}$ is of the form $\mathcal{F}_{x}:=\left\{A \in[m]^{(k)}: x \in A\right\}$ for some $x \in[m]$. Such a family is often called an EKR family in honor of the theorem. The pedestal of the EKR $\mathcal{F}_{x}$ is simply $\{\{x\}\}$.

Example 2.3. The Theorem of Hilton and Milner [5, 7] states that, for sufficiently large values of $m$, the second-biggest class of intersecting $k$-uniform families are, up to isomorphism, of the form $\mathcal{H}:=\left\{A \in[m]^{(k)}: 1 \in A ;[2, k+1] \cap A \neq \oslash\right\} \bigcup\{[2, k+1]\}$. The pedestal $P(\mathcal{H})$ is then $P(\mathcal{H})=\{\{1, j\}: j \in[2, k+1]\} \bigcup\{[2, k+1]\}$.

In words, Hilton-Milner sets are described as follows: start with an EKR family $\mathcal{F}$; adjoin a new set $X$ not in $\mathcal{F}$, and then remove from $\mathcal{F}$ all members that have empty intersection with $X$.

Remarks on $P(\mathcal{F})$ :

i In general, $P(\mathcal{F})$ is not $k$-uniform.

ii $P(\mathcal{F})$ is an antichain because of the word "minimal" in its definition.

iii If $\mathcal{F}$ is intersecting and $m \geq 2 k$, then $P(\mathcal{F})$ is intersecting. This is the only case of immediate interest, although the definition of "pedestal" doesn't depend on the fact that $\mathcal{F}$ is intersecting. 
iv If $\mathcal{F}$ is maximal, then $P(\mathcal{F})$ is maximal among those antichains in $2^{[m]}$ consisting entirely of sets of size at most $k$ (this size restriction is sometimes conveyed as $\left.P(\mathcal{F}) \subset[m]^{\leq(k)}\right)$. A finite projective plane $P G(2, q)$, is its own pedestal, i.e., that $P(P G(2, q))=P G(2, q) . P G(2, q)$ is a maximal intersecting $q+1$-regular antichain, but is not maximal in the larger class of intersecting antichains in $2^{[m]}$ [8], p. 159 .

v $P(\mathcal{F})$ omits explicit reference to $[m]$, so one may reverse the process of its construction as follows: Fix an intersecting (though not necessarily uniform) family $\mathcal{P}$, and fix an integer $k$ at least as large as the largest member of $\mathcal{P}$. For $m \geq 2 k$ we may then define the unique family $F_{m}(\mathcal{P})$ as follows: Let $J_{m}(\mathcal{P}) \in 2^{[m]}$ denote the collection of those subsets of $[m]$ containing at least one member of $\mathcal{P}$. Then $F_{m}(\mathcal{P}):=[m]^{(k)} \cap J_{m}(\mathcal{P})$ is the unique $k$-uniform family $\mathcal{F}$ of subsets of $[m]$ satisfying $P(F(\mathcal{P}))=\mathcal{P}$.

Suppose $k$ and $\mathcal{P}$ given. Define the growth function $f_{\mathcal{P}}: \mathbb{N} \rightarrow \mathbb{N}$ by the rule $f_{\mathcal{P}}(m)=$ $\left|F_{m}(\mathcal{P})\right|$. The function $f_{\mathcal{P}}$ is roughly a polynomial in $m$ with rational coefficients, because, owing to the classical theory of inclusion-exclusion, it is a finite integral combination of binomials in $m$, (c.f., e.g., [10], Chapter 2). By "roughly" we mean "ignoring conundra involving the domain of definition, as for values of $k$ too small to allow $\mathcal{P}$ to be the pedestal of a $k$-uniform family". Such conundra may be resolved by introducing an equivalence relation on $\cong$, where two functions $f_{i}: \mathbb{N} \rightarrow \mathbb{N}, i:=1,2$ may be said to satisfy $f_{1} \cong f_{2}$ if they agree on all but a finite number of terms. This formalism permits us to define the growth polynomial $p_{\mathcal{P}} \in \mathbb{Q}[m]$ to be the unique polynomial equivalent to $f_{\mathcal{P}}$. Then $p_{\mathcal{P}}$ has degree $d$ and leading coefficient $a_{d}$, where $k-d$ is the smallest size of a member of $\mathcal{P}$, and $a_{d}$ is the number of sets of size $k-d$ in $\mathcal{P}$. This affords a relatively easy way of estimating the growth of $F_{m}(\mathcal{P})$ with $m$.

Here are a few examples of growth polynomials.

i Let $\mathcal{P}:=\{\{x\}\}$, the familiar pedestal of an EKR family. Then $|F(\mathcal{P})|=\left(\begin{array}{c}m-1 \\ k-1\end{array}\right)$. This example shows why we must regard $p$ as a polynomial with rational, rather than integral coefficients. For example, when $k=3$ we get $p(m)=m^{2} / 2-3 m / 2+1$.

ii Let $\mathcal{P}:=\left\{\left\{x, y_{1}\right\},\left\{x, y_{2}\right\},\left\{y_{1}, y_{2}\right\}\right\}$. This is a sort of Hilton-Milner pedestal. In this case $p_{\mathcal{P}}=3\left(\begin{array}{c}m-2 \\ k-2\end{array}\right)-2\left(\begin{array}{c}m-3 \\ k-3\end{array}\right)$.

iii Let $\mathcal{P}:=\left\{\left\{x, y_{1}\right\},\left\{x, y_{2}\right\},\left\{x, y_{3}\right\},\left\{y_{1}, y_{2}, y_{3}\right\}\right\}$. This is another sort of Hilton-Milner pedestal. In this case again $p_{\mathcal{P}}=3\left(\begin{array}{c}m-2 \\ k-2\end{array}\right)-2\left(\begin{array}{c}m-3 \\ k-3\end{array}\right)$. This shows that it is possible for two nonisomorphic pedestals to have identical growth polynomials. In this case, the two given pedestals have different growth functions, for the trivial reason that that their unions have size 3 and 4 respectively, so $f_{\mathcal{P}}(3)$ equals zero here and one in the preceding example.

iv Given a $k$-uniform family that coincides with its pedestal, $\mathcal{P}$, the polynomial $p_{\mathcal{P}}$ is constant. We observed above that finite projective planes constitute one such set of families. 
$\mathrm{v}$ Here is another family of families each of which coincides with its pedestal. Let $n$ be an integer equal to $2 \bmod 4$. Define $\mathcal{F} \subset[n]^{(n / 2)}$ as follows: $\mathcal{F}:=\left\{A \in[n]^{(n / 2)}\right.$ : $\sum_{a \in A} a$ is odd $\}$. In words, $\mathcal{F}$ consists of those sets the sum of whose terms is odd. We argue that $\mathcal{F}$ is an intersecting family as follows: suppose to the contrary that $\mathcal{F}$ contained both a set $A$ and its complement. Then their union would be all of $[n]$, and the sum of the terms in $[n]$ would then satisfy $\sum_{a \in[n]} a$ is even. But $n=2 \bmod$ 4 , so $\sum_{a \in[n]} a$ is odd. But no $n / 2-1$-subset of $[n]$ can exhaust all of either the odd or even elements of $[n]$, so $\mathcal{F}$ is its own pedestal. Again, $p_{P(\mathcal{F})}$ is constant.

While not used in the sequel, the growth polynomial is mentioned here because it constitutes perhaps the most interesting invariant of a pedestal, and may sometimes be used in, for example, the determination of $b+1$-fold chromatic number of Kneser graphs $K_{a: b}$ for small values of $b$.

Definition 2.4. Given a family $\mathcal{F} \subseteq[m]^{(k)}$, define $S(\mathcal{F})$, the span of its pedestal, to be $S(\mathcal{F}):=\bigcup_{A \in P(\mathcal{F})} A$.

Lemma 2.5. Suppose $\mathcal{F} \subseteq[m]^{(k)}$ to be a maximal intersecting family, where $m \geq 2 k$. Then, given any $A \in P(\mathcal{F}), x \in A$, there is some $D \in P(\mathcal{F})$ such that $A \cap D=\{x\}$.

Proof. Suppose otherwise. Let $\mathcal{Y}$ be the family obtained on replacing $A$ by $A \backslash x$ in $P(\mathcal{F})$. Consider the collection of $k$-supersets of members of $\mathcal{Y}$. This collection contains $\mathcal{F}$ properly, contradicting the maximality of $\mathcal{F}$.

We can now state the principle technical result mentioned above:

Theorem 2.6. Given $k$, there is an integer $g(k)$ such that, given any natural number $m$ and any maximal intersecting family $\mathcal{F} \subseteq[m]^{(k)}$, it is true that $|S(\mathcal{F})| \leq g(k)$.

Happily, we have discovered two wholly independent proofs of this claim, which constitute the body of Section 3 of this note. The first uses Ramsey's theorem and Bollobàs's well-known Sperner-type theorem dating back to 1965. The second uses Erdös's Sunflower lemma, and yields a vastly better, though not sharp, bound. For reference, here are these three results in the required form:

Theorem 2.7. Ramsey's Theorem [2] p. 188: Let $K_{[m]}$ denote the complete graph with vertex set $[m]$. Suppose the edges of $E\left(K_{[m]}\right)$ two-colored (i.e., suppose that we are given a function $c: E\left(K_{[m]}\right) \rightarrow\left\{\right.$ red,blue\}, where $E\left(K_{[m]}\right)$ denotes the edge set of $K_{[m]}$.) Then there is a subset $S$ of the vertex set $[\mathrm{m}]$ such that:

$i S$ is at least as big as the base-4 logarithm of $m$.

ii The restriction of $c$ to the subgraph of $K_{[m]}$ induced on $S$ is constant. In other words, all edges both ends of which lie in $S$ have the same color. 
Theorem 2.8. Bollobàs's Theorem( [8], p. 102): Let $\left\{A_{1}, A_{2}, \ldots A_{t}\right\}$ be an a-uniform family of subsets of $[m]$; let $\left\{B_{1}, B_{2}, \ldots B_{t}\right\}$ be a b-uniform family of subsets of $[m]$. Suppose that $A_{i} \cap B_{j}=\oslash$ iff $i=j$. Then $t \leq\left(\begin{array}{c}a+b \\ b\end{array}\right)$.

Definition: A sunflower with $t$ petals and a core $Y$ is a family $\left\{A_{1}, A_{2}, \ldots A_{t}\right\}$ with the property that the intersection of any two of these sets is $Y$, i.e., $A_{i} \cap A_{j}=Y$ for distinct $i, j$ ( [8], p. 79). The petals are the sets $B_{i}:=A_{i} \backslash Y: i \in[t]$, and are required to be nonempty (and necessarily pairwise disjoint).

Lemma 2.9. Erdös's Sunflowers Lemma: ([8], p. 79): Let $\mathcal{F}$ be an $r$-uniform family of sets. If $|\mathcal{F}|>r !(t-1)^{r}$, then $\mathcal{F}$ contains a sunflower with $t$ petals.

\section{Proofs of Theorems}

Disclaimer: As noted above, the first proof of Theorem 2.6 relies on the theorems of Ramsey and Bollobàs, and yields a poor bound. The second proof uses Erdős's Sunflower Lemma. We follow the precedent ( [4]) of displaying Ramsey-theoretic proofs alongside more efficient proofs using other methods; the reader in a hurry should skip directly to the second proof.

\subsection{First Proof of Theorem 2.6}

Suppose we are given a maximal intersecting $k$-uniform family $\mathcal{F}$, its pedestal $P(\mathcal{F})$, and span $S(\mathcal{F})$. Define $U:=k^{2} 4^{k}$. Define $T$ to be a tower of $k$ 32's (i.e., $T:=(32)^{32 \cdots 32}$, containing $k$ instances of 32 . Suppose that $S(\mathcal{F})$ is of size at least $T^{U}$, We want to show a contradiction, the purport of which is that $S(\mathcal{F})$ can't really be that big.

The argument here involves edge-coloring a succession of complete graphs $K_{V_{t}}$, where the vertex set $V_{t}$ of the $t^{\prime}$ th graph is a collection of $t$-subsets of $S$. Begin by defining $V_{1}:=\{\{x\}: x \in S(\mathcal{F})\}$. Let $K_{V_{1}}$ be the complete graph on $V_{1}$.

We now set $t:=1$, and iterate the following argument $k$ times: Introduce a red-blue coloring on the edges of $K_{V_{t}}$ as follows:

(Coloring Step): Color the edge $\{a, b\}$ red if there is some $A \in P(\mathcal{F})$ containing $a$ and meeting $b$, or meeting $a$ and containing $b$, blue else. Note that in the case $t=1$ these two tests are indistinguishable, but otherwise not. Also observe, that, if this process lasts until $t=k$, all edges are necessarily blue, at which point things will grind to a halt as explained in (ii) below. Let $H$ be a maximum monochromatic induced subgraph of $K_{V_{t}}$. There are two cases to consider.

(i) If $H$ is red, pick a maximum matching $M$ in $H$. The purpose of the matching is to preserve the disjointness of the vertices of $K_{V_{t}}$ as we induct on $t$. Initialize $V_{t+1}=\oslash$. For each edge $e=\{a, b\}$ in the matching, do the following: We know that, since $e$ is red, either there is some $A \in P(\mathcal{F})$ containing $a$ and meeting $b$, or meeting $a$ and containing $b$. Without loss of generality, assume that we have $A \in P(\mathcal{F})$ containing $a$ and meeting b. Pick $y \in b \cap A$, and add $a \cup\{y\}$ to $V_{t+1}$. 
Observe that when we have performed this step for each edge in $M, V_{t+1}$ is a collection of pairwise disjoint $t+1$-subsets of $S(\mathcal{F})$, each of which occurs as a subset of some $A \in P(\mathcal{F})$. Also, $\left|V_{t+1}\right|=|M|=|V(H)| / 2 \geq\left\lfloor\log _{4}\left(\left|V_{t}\right|\right) / 2\right\rfloor$ by Ramsey's theorem. ( This is the reason for using a tower of big numbers in estimating the size of $V_{1}$ : at each step we take a base-4 logarithm, halve the size of what's left, and round down.)

Having defined $V_{t+1}$, define $K_{V_{t+1}}$ to be the complete graph on this vertex set, increment $t$, and return to the Coloring Step above.

(ii) If $H$ is blue, then endeavor to invoke Bollobàs's theorem mentioned above. To this end, associate with each vertex $x$ of $H$ an element $A_{x} \in P(\mathcal{F})$, where $x \in A_{x}$. In each case, pick $y_{x} \in x$, and, using Lemma 2.5 above, pick $D_{x} \in P(\mathcal{F})$ such that $A_{x} \cap D_{x}=\left\{y_{x}\right\}$. Define $B_{x}:=D_{x} \backslash y_{x}$. Consider the set set $\mathcal{W}:=\left\{A_{x}, B_{x}: x \in V(H)\right\}$.

We claim that $A_{r} \cap B_{s}=\oslash$ iff $r=s$. Here are the verifications:

a If $r=s$, then $A_{r}$ and $B_{r}$ are disjoint by construction: $B_{r}$ was constructed by removing from $D_{r}$ the one element it shared with $A_{r}$.

b if $r \neq s$, then $y_{s}$ is not an element of $A_{r}$ because the edge $\{r, s\}$ is blue in $H . A_{r}$ and $D_{s}$ meet because $P(\mathcal{F})$ is an intersecting set family. It follows that $A_{r}$ and $D_{s} \backslash y_{s}$ likewise meet.

There remains one obstacle to invocation of Bollobàs's theorem. The theorem applies only to the case where every set $A_{x}$ is of the same size $a$, and every set $B_{x}$ is of the same size $b$. However, every set in $P(\mathcal{F})$ is of size between 1 and $k$, so for each pair $(i, j) \in[k] \times[k]$ we may construct $\mathcal{W}_{(i, j)}:=\left\{A_{x}, B_{x}: x \in V(H),\left|A_{x}\right|=i,\left|B_{x}\right|=j\right\}$. Вy the pigeonhole principle and our judicious choice of $U$ in the first paragraph of this proof, there is some combination $(i, j)$ for which $\left|\mathcal{W}_{(i, j)}\right|$ violates Bollobàs's theorem, which is the desired contradiction.

\subsection{Second Proof of Theorem 2.6}

Suppose $|P(\mathcal{F})|>k !(k)^{k+1}$. Each element of $P(\mathcal{F})$ is a set of size between 1 and $k$. Partition $P(\mathcal{F})$ according to the size of its members, yielding at most $k$ families. The largest block $X$ of this partition is a uniform family containing at least $k !(k)^{k}$ members. By Erdős's Sunflower lemma, $X$ contains a sunflower $Q$ with $k+1$ petals and core $Y$. $Y$ is nonempty because $Q$ is a subset of $P(\mathcal{F})$, which is an intersecting family. Let $A$ be any member of $Q, B:=A \backslash Y$. Fix $x \in B$. By Lemma 2.5 we can find $D \in P(\mathcal{F})$ such that $A \cap D=\{x\}$. Since $P(\mathcal{F})$ is intersecting, $D$ must also meet each of the $k$ remaining

members of $Q$. However, since $A \cap D=\{x\}, Y \cap D=\oslash$. It follows that $D$ must contain at least one element from each of the $k+1$ pairwise disjoint petals of $Q$. But $|D| \leq k$, a contradiction.

\subsection{Proof of Theorem $\mathbf{1 . 2}$}

$|S(\mathcal{F})| \leq g(k)$ by Theorem 2.6; this is the ground set on which $P(\mathcal{F})$ is defined. Each set in $P(\mathcal{F})$ has size at most $k$. The number of sets of size at most $k$ defined on this ground 
set is $T:=\sum_{i \in[k]}\left(\begin{array}{c}g(k) \\ i\end{array}\right)<2^{g(k}$. Since $P(\mathcal{F})$ is an intersecting family, its size is bounded by the EKR Theorem, and satisfies $U:=|P(\mathcal{F})| \leq\left(\begin{array}{c}g(k)-1 \\ k-1\end{array}\right)$. It follows that the number of ways in which $P(\mathcal{F})$ can be defined is bounded above by $\left(\begin{array}{l}T \\ U\end{array}\right)<2^{T}$. The number of distinct isomorphism classes is bounded by this number.

This upper bound may be lowered greatly in various ways as follows:

i Almost all hypergraphs are rigid, so one can divide by a factor of about $g(k)$ ! It would take some work, here deferred, to make this claim wholly precise.

ii The Sunflower Lemma does not stipulate that the family in question $(P(\mathcal{F})$ here $)$ is intersecting. Stronger variants are available for intersecting families, improving the bound on $g(k)$ by a factor of about $k$.

\section{Generalizations}

The results presented so far concern set systems, and may be couched in the language of Boolean Lattices. Some of these results admit generalizations to larger classes of lattices. The goal of this section is to present these generalizations. Suppose $L$ to be an arbitrary lattice. Given $\mathcal{F} \subset L$, we say that $\mathcal{F}$ is intersecting if the meet of any two elements $A, B \in \mathcal{F}$ satisfies $A \wedge B>\hat{0}_{L}$. In the case where $L$ is a Boolean Lattice, this coincides with the usual stipulation that $F$ be an intersecting family.

By a graded lattice we mean a lattice each of whose elements has a well-defined rank. In a graded lattice $L$, we may say that $\mathcal{F} \subset L$ is $k$-uniform if the rank of each $A \in \mathcal{F}$ is $k$. Thus, the notion of a maximal intersecting $k$-uniform family in an arbitrary graded lattice $L$ is clear: it is simply a set $\mathcal{F} \subset L$ which is maximal with respect to the property that it is both intersecting and $k$-uniform. All lattices considered here are finite. Recall that in

To permit the definition of "pedestal" in this more general setting, we introduce the following notation: given $a \in L$ of rank at most $k$, let $U(a) \subset L$ denote the set of all elements of $L$ of rank $k$ dominating $a$. In this more general setting, given a $k$-uniform family $\mathcal{F}$, define its prepedestal as $P P(\mathcal{F}):=\{a \in L: U(a) \subset F\}$, and define the pedestal of $\mathcal{F}$ to be the set of minimal elements of the prepedestal.

Two families $\mathcal{F}_{1}, \mathcal{F}_{2} \subset L$ are declared isomorphic if each lies in the orbit of the other under the action of $\operatorname{Aut}(L)$, the automorphism group of $L$. For arbitrary lattices it may happen that $\operatorname{Aut}(L)$ is trivial, in which case it is difficult to say much. Indeed, in a rigid lattice we may construct a large family of pairwise nonisomorphic maximal intersecting 1-uniform families as follows: identify with each atom $a \in L$ the set $\{a\}$; the set of such sets is then a collection of pairwise nonisomorphic families. To avoid this sort of problem, we define a homogeneous lattice to be one whose automorphism group acts on the set $A \subset L: A=\{a \in L, a$ is an atom $\}$ as the symmetric group $S_{A}$.

While most of the preliminary results stated in this section hold for more general classes of lattices, we confine the statement of these claims to the class of finite homogeneous distributive lattices, which we refer to as admissible lattices. Some of the arguments above, 
such as the EKR theorem, required that the ground set be large enough. The analogous stipulation here is that, given $k$, we say that an admissible lattice $L$ is $k$-admissible if, given any $a \in L$ of rank $k$, there is some $b \in L$ of rank $k$ such that $a \wedge b=0$.

It transpires [9] that the Sunflower lemma, the main tool of the last section, holds for a large class of lattices, including distributive lattices. Indeed, for each $k$, essentially all the results of this paper hold for the class of $k$-admissible lattices.

Lemma 4.1. Let $k$ be an integer, and let $L$ be a $k$-admissible lattice. Suppose $\mathcal{F} \subset L$ is a maximal intersecting $k$-uniform family. Then any $a \in P(\mathcal{F})$ is a join of atoms in $L$.

Proof. Suppose otherwise. Let $b \in P(\mathcal{F})$, and let $b^{*}$ denote the join of all atoms dominated by $b$. Suppose $b \neq b^{*}$. Then $b^{*}<b$, so we consider the effect of replacing $b$ by $b^{*}$ in $P(\mathcal{F})$. Now, since $\mathcal{F}$ is intersecting, any $x$ in $\mathcal{F}$ satisfies $x \wedge b>\hat{0}_{L}$. But then $x$ must dominate some atom lying below $b$, so $x \wedge b^{*}>\hat{0}_{L}$ as well. Conversely, let $y \in U\left(b^{*}\right)$. Then $y$ dominates all the atoms lying beneath $b$, so $y$ meets each $x$ in $\mathcal{F}$. We arrive at a contradiction: replacement of $b$ by $b^{*}$ in $P(\mathcal{F})$ yields an intersecting family at least as large as $\mathcal{F}$, so either $\mathcal{F}$ isn't maximal or $b$ isn't minimal.

The preceding lemma allows us to repeat most of the arguments of the preceding sections, because, given any family $F$ we can now simply identify each element $A$ of its pedestal with the set of atoms dominated by $A$. In particular, we define the span $S(\mathcal{F})$ of the pedestal to be the union of these sets of atoms, so $S(\mathcal{F})$ is likewise a set of atoms of the ambient lattice.

We now state the principal results of the preceding sections in lattice form, noting that the proofs are unaltered.

Lemma 4.2. Suppose $\mathcal{F}$ to be a maximal $k$-uniform intersecting family in a $k$-admissible lattice $L$. Then, given any $A \in P(\mathcal{F}), x$ an atom of $L$ dominated by $A$, there is some $D \in P(\mathcal{F})$ such that $A \cap D=\{x\}$.

Theorem 4.3. Given $k$, there is an integer $g(k)$ such that, given any $k$-admissible lattice $L$ and any maximal $k$-uniform intersecting family $\mathcal{F} \subset L$, it is true that $|S(\mathcal{F})| \leq g(k)$.

Theorem 4.4. The number of pairwise nonisomorphic maximal intersecting $k$-uniform families of subsets of a $k$-admissible lattice $L$ is bounded by a constant $f(k)$ not depending on $L$.

\section{Concluding Remarks}

As usual, Ramsey theory is effective at proving the existence of an upper bound, but not of providing a sharp upper bound. The author suspects that the true value of $g(k)$ is $k^{2}-k+1$, given by the finite projective planes. In case the $k=3$, the author has verified that all maximal intersecting families defined on a ground set of less than 12 elements satisfy the condition that the size of the span is at most $3^{2}-3+1=7$. Indeed, there appear to be only 15 distinct pairwise nonisomorphic pedestals for $k \leq 3$, viz: 
The gap between this experimental bound of 15 and that assured by the theorem above is nothing if not large.

Not treated here is the expected growth of the "length" $L(P(\mathcal{F})):=|P(\mathcal{F})|$. The author supposes this to be strictly exponential in $k$, i.e., one guesses that there exist real numbers $a, b$ such that $a^{k}<E[L(P(\mathcal{F}))]<b^{k}$.

A finite projective plane $P G(2, q)$ is its own pedestal. There are many other families with this property, but the author does not know whether almost all families have this property.

The number of pairwise nonisomorphic $k$-uniform intersecting families admits a superexponential lower bound, as the following argument shows. For any natural number $k$, consider the $h(k):=\left(\begin{array}{c}2 k \\ k\end{array}\right) / 2$ ways of partitioning $[2 k]$ into two subsets of equal size. Evidently there are $2^{h(k)}$ ways to select one block from each of these partitions. For $m>2 k$, each such selection $S$ may be extended to a maximal $k$-uniform family $S^{\prime}$ in one or more ways. The point is that distinct selections $S, T$ give rise to distinct maximal intersecting extensions. Thus, even when $k=4$, this yields a computationally daunting number of distinct families (at least $2^{35}$ ). Note that $k !=o\left(2^{h(k)}\right)$, so the computation of isomorphism classes of maximal intersecting $k$-uniform families is likewise daunting.

\section{References}

[1] Bela Bollobàs, Combinatorics: Set Systems, Hypergraphs, Families of Vectors, and Combinatorial Probability, Cambridge University Press 1986.

[2] R. Diestel, Graph Theory, Springer-Verlag 1997.

[3] P. Erdős, C. Ko, and R. Rado, "Intersection theorems for systems of finite sets," Quart. J. Math. Oxford, vol. 12, pp. 313-320, 1961 
[4] P. Erdős and G. Szekeres, "A combinatorial problem in geometry", Comp. Math. 2 (1935), 464-470.

[5] P. Frankl, "An extremal problem for two families of sets", European Journal of Combinatorics 3, 1982, 125-127.

[6] P.Frankl, "The Shifting Technique in Extremal Set Theory", in Surveys in Combinatorics 1987, ed. C. Whitehead, Cambridge University Press 1987, pp 81-110.

[7] A.J.W. Hilton, E. C. Milner, Some intersection theorems for systems of Finite sets, Quart. J. Math. Oxford (2) 18 (1967), 369-384.

[8] S. Jukna, Extremal Combinatorics, Springer 2001

[9] G. McKenna, Sunflowers in Lattices, submitted.

[10] R. Stanley, Enumerative Combinatorics, vol. 1, Wadsworth and Brooks/Cole, Pacific Grove, CA, 1986 